LU TP 01-38

hep-ph/0112284

December 2001

Revised May 2002

\title{
Correcting the Colour-Dipole Cascade Model with Fixed Order Matrix Elements
}

\author{
Leif Lönnblad \\ Dept. of Theoretical Physics, \\ Sölvegatan 14A, S-223 62 Lund, Sweden \\ Leif.Lonnblad@thep.lu.se
}

\begin{abstract}
An algorithm is presented in which the Colour-Dipole Cascade Model as implemented in the ARIADNE program is corrected to match the fixed order tree-level matrix elements for $\mathrm{e}^{+} \mathrm{e}^{-} \rightarrow n$ jets. The result is a full parton level generator for $\mathrm{e}^{+} \mathrm{e}^{-}$annihilation where the generated states are correct on tree-level to fixed order in $\alpha_{\mathrm{s}}$ and to all orders with modified leading logarithmic (MLLA) accuracy. In this paper, matrix elements are used up to second order in $\alpha_{\mathrm{s}}$, but the scheme is applicable also for higher orders. A strategy for also including exact virtual corrections to fixed order is suggested and the possibility to extend the scheme to hadronic collisions is discussed.
\end{abstract}




\section{Introduction}

Perturbative QCD has been very successful in describing many features of multiparticle production in high energy collisions. There are, however, several problems which have not yet been solved, mostly related to the transition between the perturbative and non-perturbative description of the theory. Observables involving a few widely separated jets are in principle well described with fixed-order perturbative matrix elements (MEs) for producing a few partons. But to make precision comparisons with experiments, it is important to understand the transition of these partons to observable hadrons. Our best knowledge of this transition comes from hadronization models which describes how multi-parton states are transformed into multi-hadron ones. But for these models to work reliably one needs also a description of the collinear and soft partons describing the internal structure of widely separated jets and the soft partons between the jets.

To describe soft and collinear partons it is not feasible to use fixed-order perturbation theory. Not only do the MEs for many-parton states become extremely complicated but, since the partons are no longer widely separated, the increase in phase space introduces large logarithms which compensates the smallness of $\alpha_{\mathrm{s}}$ and makes the whole perturbative expansion ill-behaved. To describe the inner structure of jets, a more practical approach is to use a parton shower (PS) procedure. Here the large logarithms are resummed to all orders at the expense of only keeping the leading logarithmic behaviour of the full matrix elements.

To get a near complete description of multi-particle production it would be desirable to combine the generation of a few widely separated partons according to fixed-order MEs with the evolution of these states according PSs and finally the transition into hadrons using a hadronization model. To do this is, however, highly non-trivial and so far there exist no general procedure which is entirely satisfactory. The main problem is that one needs a resolution scale to separate the ME generation from the PS one. This scale needs to be small enough to benefit from the full ME description, but if it becomes too small the final result is spoiled by non-physical large logarithms involving the separation scale.

For some special cases such as e.g. $\mathrm{e}^{+} \mathrm{e}^{-} \rightarrow 3$ jets [1] 6], $\mathrm{e}^{-} \mathrm{p} \rightarrow 2+1$ jets [7, 8] and $\mathrm{pp} \rightarrow \mathrm{W}+1$ jet [9 12], there are working procedures for combining ME and PS. Recently there has also been suggested a couple of more general procedures [13 18], but none of them can be considered to be the final word on the matter. In this paper the procedure by Catani and collaborators in 16] is taken as a starting point to create a matching between fixed order $\mathrm{ME}$ generators and the colourdipole cascade model (CDM) [1, 19] as implemented in the ARIADNE [a] event generator. The resulting algorithm is also not complete, but it provides a cleaner interface between the ME and PS stages of the generation, and it carefully treats 
the influence of the available phase space on the Sudakov form factors needed to correct the ME generation.

The outline of this paper is as follows. In section 1 the algorithm of Catani et al. is presented, reformulated to better fit the CDM. Then, in section 3, the reconstruction of the resolution scales of a partonic state generated with MEs is discussed, followed by the description in section 1 of how the Sudakov form factors are calculated and a step by step description of the whole algorithm in section 5. In section

6 a few initial results from the new algorithm for $\mathrm{e}^{+} \mathrm{e}^{-} \rightarrow n$ jets with $n \leq 4$ are presented. Finally, in section 0, some conclusions are presented together with a discussion on how the procedure can be extended to also be used for collisions involving hadrons in the initial state. Also the possibility to improve the description to get virtual corrections exact to fixed-order perturbation theory is discussed.

\section{Matrix elements and parton cascades}

To describe $\mathrm{e}^{+} \mathrm{e}^{-}$annihilation into $n$ jets with fixed order perturbation theory one needs to introduce a resolution scale to avoid divergencies in the ME. This scale can be defined in many different ways, usually connected to a specific jet reconstruction algorithm which can be used both on the $\mathrm{ME}$ and on the finalstate hadrons observed in an experiment. Given such a resolution scale $Q_{0}$ and a maximum scale, $Q$, set by the total center of mass energy, we can write down the fraction of $n$ jet events for $n \leq 4, R_{n}\left(Q, Q_{0}\right)$, given by the second order MEs as

$$
\begin{aligned}
& R_{2}\left(Q, Q_{0}\right)=1+\alpha_{\mathrm{s}} C_{0,1}\left(Q, Q_{0}\right)+\alpha_{\mathrm{s}}^{2} C_{0,2}\left(Q, Q_{0}\right) \\
& R_{3}\left(Q, Q_{0}\right)=\alpha_{\mathrm{s}} C_{1,1}\left(Q, Q_{0}\right)\left(1+\alpha_{\mathrm{s}} C_{1,2}\left(Q, Q_{0}\right)\right) \\
& R_{4}\left(Q, Q_{0}\right)=\alpha_{\mathrm{s}}^{2} C_{2,2}\left(Q, Q_{0}\right)
\end{aligned}
$$

where $R_{4}$ receives contributions from $q \bar{q} g g$ as well as from $q \bar{q} q^{\prime} \bar{q}^{\prime}$ final states. The coefficients $C_{n, m}\left(Q, Q_{0}\right)$ are related to the emission of $n$ partons to $\mathcal{O}\left(\alpha_{\mathrm{s}}^{m}\right)$, i.e. $C_{n, n}$ corresponds to resolved tree-level diagrams and $C_{n, m}$, with $m>n$, corresponds to the sum of virtual diagrams and unresolved emission diagrams. The problem with a small resolution scale comes about because the coefficients contains logarithms of $Q / Q_{0}$ which, for small $Q_{0}$, destroys the $\alpha_{\mathrm{s}}$-expansion. In fact $R_{3}$ above becomes negative for small enough $Q_{0}$.

The parton shower approach is quite different. Here gluons are emitted or split into $q \bar{q}$ pairs in according to simple $1 \rightarrow 2$ (or, as in the CDM case, $2 \rightarrow 3$ ) splitting functions in an ordered probabilistic cascade. The emissions are hence ordered according to an associated evolution scale so that the first emissions has the highest scale and subsequent emissions decreasingly lower scales. The definition of the evolution scale, $\rho$, is different in different shower models, e.g. invariant mass 
in PyThiA [20] and invariant transverse momentum in ARIADNE, but common for all models is the occurrence of a Sudakov form factor corresponding to the probability of having no emissions between two given scales. If $\alpha_{\mathbf{s}} \mathcal{P}(\rho, z, \phi)$ is the splitting probability for emitting a gluon from the initial $q \bar{q}$ pair, the two-jet rate above some scale $\rho_{0}$ becomes

$$
R_{2}\left(\rho_{m}, \rho_{0}\right)=\Delta_{S_{2}}\left(\rho_{m}, \rho_{0}\right)=\exp \left(-\alpha_{\mathrm{s}} \int_{\rho_{0}}^{\rho_{m}} d \rho \int d z \int d \phi \mathcal{P}(\rho, z, \phi)\right)
$$

where $\rho_{m}$ is the maximum kinematically allowed scale, and the $z$ and $\phi$ integrals are over all kinematically allowed values. Performing the integrals and expanding the exponential, the two-jet rate can be expressed in the same form as the matrix element in eq. (11)

$$
R_{2}\left(\rho_{m}, \rho_{0}\right)=1+\alpha_{\mathrm{s}} C_{0,1}^{\mathrm{PS}}\left(\rho_{,} \rho_{0}\right)+\alpha_{\mathrm{s}}^{2} C_{0,2}^{\mathrm{PS}}\left(\rho_{m}, \rho_{0}\right)+\ldots
$$

Similarly the three-jet rate according to a parton shower scenario can be written as

$$
R_{3}\left(\rho_{m}, \rho_{0}\right)=\alpha_{\mathrm{s}} \int_{\rho_{0}}^{\rho_{m}} d \rho \int d z \int d \phi \mathcal{P}(\rho, z, \phi) \Delta_{S_{2}}\left(\rho_{m}, \rho\right) \Delta_{S_{3}}\left(\rho, \rho_{0}\right),
$$

i.e. the probability of emitting a gluon at some scale $\rho$, times the probability that no gluon was emitted above (before) $\rho$ and the probability that nothing was emitted below. The latter is also a Sudakov form factor, symbolically written $\Delta_{S_{3}}\left(\rho, \rho_{0}\right)$, giving the probability of not having any further emissions from the qg $\bar{q}$ state above the scale $\rho_{0}$ and will in general depends on the full kinematics of the three-parton state. Still, it can be written as an exponential of minus an emission probability integrated over the allowed phase space. It is therefore easily expanded in powers of $\alpha_{\mathrm{s}}$ and, after integrating an expression similar to the one in eq. (11) can be obtained:

$$
R_{3}\left(\rho_{m}, \rho_{0}\right)=\alpha_{\mathrm{s}} C_{1,1}^{\mathrm{PS}}\left(\rho_{,} \rho_{0}\right)\left(1+\alpha_{\mathrm{s}} C_{1,2}^{\mathrm{PS}}\left(\rho_{m}, \rho_{0}\right)+\ldots\right) .
$$

Note that, as in eq. (11), the coefficient $C_{1,2}^{\text {PS }}$ can become large and negative, but since it is the result of an expansion of an exponential which is always positive and below one, the $R_{3}$ will always be positive and finite.

For the four-jet rate there is an additional complication since there are both qquggand $q \bar{q} q^{\prime} \bar{q}^{\prime}$-states, but it can be written on the form

$$
\begin{aligned}
& R_{4}\left(\rho_{m}, \rho_{0}\right)=\alpha_{\mathrm{s}}^{2} \int_{\rho_{0}}^{\rho_{m}} d \rho_{1} \int d z_{1} \int d \phi_{1} \mathcal{P}\left(\rho_{1}, z_{1}, \phi_{1}\right) \Delta_{S_{2}}\left(\rho_{m}, \rho_{1}\right) \Delta_{S_{3}}\left(\rho_{1}, \rho_{2}\right) \times \\
& \int_{\rho_{0}}^{\rho_{1}} d \rho_{2} \int d z_{2} \int d \phi_{2}\left[\mathcal{P}_{\mathrm{gg}}\left(\rho_{2}, z_{2}, \phi_{2}\right) \Delta_{S_{\mathrm{qgg} \bar{q}}}\left(\rho_{2}, \rho_{0}\right)\right. \\
& \left.+\mathcal{P}_{\mathrm{q} \overline{\mathrm{q}}}\left(\rho_{2}, z_{2}, \phi_{2}\right) \Delta_{S_{\mathrm{q} \overline{\mathrm{q}} \mathrm{q}^{\prime} \overline{\mathrm{q}}^{\prime}}}\left(\rho_{2}, \rho_{0}\right)\right],
\end{aligned}
$$

where $\mathcal{P}_{\text {gg }}$ is the splitting function for emitting an additional gluon from the qg $\bar{q}$ state and $\mathcal{P}_{\mathrm{q} \overline{\mathrm{q}}}$ is the splitting function for splitting the gluon in the qg $\overline{\mathrm{q}}$ state into a new quark-antiquark pair. 
Again, the Sudakovs, $\Delta_{S_{\mathrm{ggg} \bar{q}}}$ and $\Delta_{S_{\mathrm{q} \overline{\mathrm{q}} q^{\prime} \bar{q}^{\prime}}}$, gives the no-emission probability from the qgg $\bar{q}$ and $q \bar{q} q ' \bar{q}$ ' states respectively and can be written as simple exponentials, which can be expanded in $\alpha_{\mathrm{s}}$ and, after integration, an expression similar to the one in eq. (1) can be written

$$
R_{4}\left(\rho_{m}, \rho_{0}\right)=\alpha_{\mathrm{s}}^{2} C_{2,2}^{\mathrm{PS}}\left(\rho, \rho_{0}\right)\left(1+\alpha_{\mathrm{s}} C_{2,3}^{\mathrm{PS}}\left(\rho_{m}, \rho_{0}\right)+\ldots\right) .
$$

The coefficients $C_{n, m}^{\mathrm{PS}}$ contains only the leading logarithmic partsf of the corresponding exact ones, $C_{n, m}$. To arrive at a combined parton shower and matrix element generator would then mean using the parton shower method but replacing the first few coefficients $C_{n, m}^{\mathrm{PS}}$ with the exact ones. Here, a more modest approach will be taken, where only the coefficients $C_{n, n}^{\mathrm{PS}}$, corresponding to the resolved treelevel diagrams will be replaced by the exact versions. This amounts to generating events according to the $n$-jet rates

$$
\begin{aligned}
& R_{2}\left(\rho_{m}, \rho_{0}\right)=\Delta_{S_{2}}\left(\rho_{m}, \rho_{0}\right), \\
& R_{3}\left(\rho_{m}, \rho_{0}\right)=\alpha_{\mathrm{s}} \int_{\rho_{0}}^{\rho_{m}} d \rho c_{1,1}^{\mathrm{ME}}\left(\rho_{m}, \rho\right) \Delta_{S_{2}}\left(\rho_{m}, \rho\right) \Delta_{S_{3}}\left(\rho, \rho_{0}\right), \\
& R_{\mathrm{qgg \overline {q }}}\left(\rho_{m}, \rho_{0}\right)=\alpha_{\mathrm{s}}^{2} \int_{\rho_{0}}^{\rho_{m}} d \rho_{1} \int_{\rho_{0}}^{\rho_{1}} d \rho_{2} c_{2,2(\mathrm{qgg} \overline{\mathrm{q}})}^{\mathrm{ME}}\left(\rho_{m}, \rho_{1}, \rho_{2}\right) \times \\
& \Delta_{S_{2}}\left(\rho_{m}, \rho_{1}\right) \Delta_{S_{3}}\left(\rho_{1}, \rho_{2}\right) \Delta_{S_{\mathrm{qgg}}}\left(\rho_{2}, \rho_{0}\right), \\
& R_{\mathrm{q} \overline{\mathrm{q}} \mathrm{q}^{\prime} \overline{\mathrm{q}}^{\prime}}\left(\rho_{m}, \rho_{0}\right)=\alpha_{\mathrm{s}}^{2} \int_{\rho_{0}}^{\rho_{m}} d \rho_{1} \int_{\rho_{0}}^{\rho_{1}} d \rho_{2} c_{2,2\left(\mathrm{q} \overline{\mathrm{q}} \mathrm{q}^{\prime} \overline{\mathrm{q}}^{\prime}\right)}^{\mathrm{ME}}\left(\rho_{m}, \rho_{1}, \rho_{2}\right) \times \\
& \Delta_{S_{2}}\left(\rho_{m}, \rho_{1}\right) \Delta_{S_{3}}\left(\rho_{1}, \rho_{2}\right) \Delta_{S_{\mathrm{q}_{\bar{q}} \bar{q}^{\prime} \bar{q}^{\prime}}}\left(\rho_{2}, \rho_{0}\right),
\end{aligned}
$$

where the $c_{n, n}^{\mathrm{ME}}$ are the differential full tree-level matrix elements (the $z$ and $\phi$ integrals have been left out for brevity).

The procedure is then to use a matrix element generator to generate exclusive $n$-parton final states, to reweight these states with the Sudakov form factors, and then add a parton cascade.

Note, however, that the $\rho$ scales are defined in a particular parton shower scenario and have no equivalents in the matrix element generation, where the notion of one emission being performed before another is not well defined. Instead these scales need to be reconstructed from the generated $n$-parton state.

In reference [16] this is achieved by using the $k_{\perp}$-algorithm [24, 25], the Sudakov form factors are then calculated according to an analytical form, and the angularordered parton shower in HERWIG [26] is added with a special veto procedure. The reason for this veto procedure is to avoid double counting, since the reconstructed

\footnotetext{
${ }^{1}$ In fact, most parton shower implementations also contain some non-leading terms corresponding to the modified leading logarithmic approximation (MLLA) 21 23]. In particular, for the case of the ARIADNE program, $C_{1,1}^{\mathrm{PS}}=C_{1,1}$ and $C_{0,1}^{\mathrm{PS}}=C_{0,1}$
} 
scales do not correspond to the ordering variable in the parton shower. The angular ordering means that one can have a soft emission at a large angle, followed by a harder emission at a smaller angle.

Here the Colour Dipole Model implemented in the ARIADNE program will be used for the parton cascade. The ordering variable is an invariant transverse momentum, so the emissions are ordered in scale. In addition, a modified version of the DICLUS 27,28 jet clustering algorithm will be used to reconstruct the emission scales given as the same invariant transverse momenta. In this way many of the problems in ref. [16] are completely avoided. The DICLUS algorithm will not only be used to reconstruct the emission scales, but to reconstruct a complete dipole cascade history for the $n$-parton states generated according to the matrix element, i.e. also the intermediate states which would occur in a dipole shower producing the same state.

These states will be used to evaluate the Sudakov form factors in exactly the same way as they are evaluated in the dipole cascade as explained in section 4 . In the procedure of Catani et al. the Sudakovs are calculated analytically, which means that they do not exactly correspond to a no-emission probability since they do not take into account that the available phase space for emission off a quark after it has emitted a gluon is changed due to recoils. In the dipole cascade the Sudakovs are generated dynamically and takes into account the actual phase space available for further emissions. To MLLA accuracy the two approaches are, however, equivalent.

\section{Reconstruction of emissions}

The colour-dipole cascade model [1, 19] describes the emission of a gluon in terms of dipole radiation from a colour dipole between two partons. The emissions are hence described as two partons going to three, rather than one going to two as in conventional parton shower models. This means that gluon coherence is automatically taken into account and that the first gluon emission in $\mathrm{e}^{+} \mathrm{e}^{-}$annihilation trivially reproduces the full first order matrix element. But there are a couple of technical details which are particular to the dipole cascade. All partons are always on-shell at each step of the cascade. The conservation of energy and momentum is achieved since both emitting partons receives a recoil from the emitted gluon. The splitting of a gluon into a $q \bar{q}$-pair is also treated as if emitted from one of the dipoles connected to the gluon, and the parton in the other end will receive some recoil in order to conserve anergy and momentum [29]. Further more, the scale of an emission is defined in terms of a Lorentz-invariant $p_{\perp}$ of the emitted parton with respect to the emitting ones. This is defined as

$$
p_{\perp}^{2}=\frac{\left(s_{12}-\left(m_{1}+m_{2}\right)^{2}\right)\left(s_{23}-\left(m_{2}+m_{3}\right)^{2}\right)}{s_{123}},
$$


where parton 2 is the emitted one and $s_{i j}$ and $s_{i j k}$ are the squared invariant masses of the two- and three-parton combinations.

The dipole clustering algorithm (DICLUS) 27,28 can be thought of as the inverse of the dipole cascade. In each step the combination of three jets which have the smallest invariant $p_{\perp}$ are clustered together into two (massless) jets. In the procedure presented here, the dipole clustering will be used with a couple of modifications. First of all the information available from the generation of the few-parton state will be used. Hence a gluon is only considered to have been emitted from the two partons to which it is colour connected 1 . So for a colour connected $\mathrm{qg}_{1} \mathrm{~g}_{2} \overline{\mathrm{q}}$ state the only possible clusterings are $\mathrm{qg}_{1} \mathrm{~g}_{2} \rightarrow \mathrm{qg}_{2}$ and $\mathrm{g}_{1} \mathrm{~g}_{2} \overline{\mathrm{q}} \rightarrow \mathrm{g}_{1} \overline{\mathrm{q}}$. Also, a $\mathrm{q} \overline{\mathrm{q}}-\mathrm{pair}$ is reconstructed into a gluon which is made massless by also considering one of the partons connected to the $\mathrm{q}$ or $\overline{\mathrm{q}}$. In this way all partons in the reconstructed states are always kept on-shell. A qq̄ pair is only reconstructed to a gluon if they are not colour-connected (directly or indirectly).

When reconstructing a $2 \rightarrow 3$ emission there is an ambiguity in the directions of the emitting partons. If the three partons are transformed into their center of mass system, the momenta of the emitting partons are easily obtained from energy momentum conservation. It is also clear that the two partons should lie in the same plane as the original three. But the angular orientation in this plane is not determined. The inverse problem is encountered in the dipole cascade where the amount of transverse recoil taken by each of the emitting partons is not given by the theory. There are different choices made for each kind of emission. In ARIADNE the choices are as follows:

- For a gluon emission from a q $\bar{q}$ dipole, one of the quarks retains its direction with a probability proportional to the square of its energy (according to the prescription in [31]).

- For gluon emission from a quark-gluon dipole, the gluon retains its direction.

- For gluon emission from a gluon-gluon dipole, the transverse recoil is shared among the emitters so that the sum of their squared transverse momenta is minimized.

- For a gluon splitting into a q $\bar{q}$ pair, the spectator parton retains its direction.

Of these, all but the first are completely deterministic and can easily be inverted and be used in the reconstruction algorithm. For the first, the prescription for gluon-gluon dipoles is followed instead [?

\footnotetext{
${ }^{2}$ This requires that the ME generator produces states with a definite colour topology. Although this information is not physical, it is usually provided by ME generator programs. See discussion in ref. [30]

${ }^{3}$ This will not influence the results in this paper where only $\alpha_{\mathrm{s}}^{2}$ ME will be used and all $\mathrm{qg} \overline{\mathrm{q}} \rightarrow \mathrm{q} \overline{\mathrm{q}}$ reconstructions will give the initial $\mathrm{q} \overline{\mathrm{q}}$ state where the angular orientation is irrelevant for the subsequent cascade.
} 
Rather than always selecting the three parton configuration which has the smallest invariant $p_{\perp}$ to be reconstructed, as is customary in jet algorithms, it is possible to reconstruct all possible dipole cascade histories. This is feasible since we are dealing with only a handful partons. The procedure will then be to choose randomly between these different histories weighted with the corresponding dipole splitting probabilities in analogy to the strategy in 13. The splitting probabilities will not include a running $\alpha_{\mathrm{s}}$ as in the normal dipole cascade, since a constant $\alpha_{\mathrm{s}}$ was used in the generation of the parton state. The running of $\alpha_{\mathrm{s}}$ will be corrected for at a later stage.

It should be noted that some of the histories may consist of sequences of un-ordered emissions which cannot have been produced by the dipole cascade, and these are excluded from the histories to choose between. In rare cases it is possible that no history can be found which correspond to an ordered sequence of dipole emissions. In this case one of the "impossible" histories are chosen, but the reconstructed scales are modified so that if the scale of one emission is smaller than the scale of the subsequent one, the larger scale is chosen for both reconstructed emissions.

With this procedure it is now possible to reconstruct a dipole cascade history for any $n$-parton state. All intermediate $2,3, \ldots, n-1$ parton states are reconstructed together with the corresponding emission scales $p_{\perp 1}^{2}, \ldots, p_{\perp n-2}^{2}$. The reconstructed scales can then be used to correct the MEs for the running of $\alpha_{\mathrm{s}}$ by rejecting the state with a probability

$$
1-\frac{1}{\alpha_{\mathrm{s} 0}^{n-2}} \Pi_{i=1}^{n-2} \alpha_{\mathrm{s}}\left(p_{\perp i}^{2}\right)
$$

where the $\alpha_{\mathrm{s} 0}$ used in the ME generation is taken at the cutoff scale $p_{\perp c}^{2}$ of the parton cascadef to ensure that the probability is positive.

\section{The Sudakov Veto algorithm}

The reconstructed scales and states are also used to calculate the correction for the Sudakov form factors. Rather than using the approximate analytic expression as a weight, we can use the fact that it corresponds to the no-emission probability in a specific region of phase space.

Consider a three-parton state generated with the $\mathcal{O}\left(\alpha_{\mathrm{s}}\right)$ ME, where the scale of the gluon emission has been reconstructed to $p_{\perp 1}^{2}$. The Sudakov form factor is then the probability of there being no emission from the initial q $\bar{q}$ state before the gluon was emitted, i.e. at a scale above $p_{\perp 1}^{2}$, and that there is no emission from the qga state between the scale $p_{\perp 1}^{2}$ and the cutoff in the ME. By making two trial

\footnotetext{
${ }^{4}$ Note that the $Q_{0}$ cut used in the ME generation is not necessarily the smallest possible invariant $p_{\perp}$ scale.
} 
emissions with the dipole cascade, one from the reconstructed $q \bar{q}$ state, starting from the maximum scale, and one from the ME-generated qg $\bar{q}$ state starting from

$p_{\perp 1}^{2}$ and rejecting the whole event if the first was at a scale above $p_{\perp 1}^{2}$ or the second was inside the ME cutoff, the probability of accepting the event is exactly equal to the Sudakov form factor. With this veto procedure the proper phase space region is taken into account rather than the approximate limits in the analytic form.

Special care must also be taken when a partonic state has been generated to the highest order in $\alpha_{\mathrm{s}}$ used for the ME generation. In that case we want to continue generating with the dipole cascade from the last reconstructed scale irrespectively of the cutoff in the MEs and we should keep the trial emission from the ME generated state and continue the cascade rather than vetoing the whole event. This corresponds to changing the four-jet rates in (8) to four-or-more-jets rates, e.g.

$$
\begin{array}{r}
R_{4 g g+}\left(\rho_{m}, \rho_{0}\right)=\alpha_{\mathrm{s}}^{2} \int_{\rho_{0}}^{\rho_{m}} d \rho_{1} \int_{\rho_{0}}^{\rho_{1}} d \rho_{2} c_{2,2(g g)}^{\mathrm{ME}}\left(\rho_{m}, \rho_{1}, \rho_{2}\right) \times \\
\Delta_{S_{2}}\left(\rho_{m}, \rho_{1}\right) \Delta_{S_{3}}\left(\rho_{1}, \rho_{2}\right) .
\end{array}
$$

This cannot easily be achieved in the procedure of Catani et al., since the angular ordering of the parton shower in HERWIG, allows for subsequent harder emissions which could result in double counting.

\section{$5 \quad$ The algorithm step by step}

We now have all the ingredients to present the whole algorithm step by step. We assume there is a matrix element generator which can generate complete partonic states according to the exact tree-level MEs up to $\mathcal{O}\left(\alpha_{\mathrm{s}}^{N}\right)$. This generator is regulated by a cutoff $Q_{0}$ to avoid divergencies. In principle this cutoff could be in the same invariant $p_{\perp}$ used as evolution variable in the dipole cascade, but to be completely general we assume that it is instead e.g. a simple cutoff in invariant mass of any two outgoing partons. This is allowed as long as there is no double counting (or under counting due to empty phase space regions) as is demonstrated below. This matrix element generator should then be combined with the standard dipole cascade of ARIADNE which has a lower cutoff in the invariant $p_{\perp}$ given by

$p_{\perp c}$. The constant $\alpha_{\mathrm{s} 0}$ used in the ME generator is taken at the scale $p_{\perp c}^{2}$ using the same $\Lambda_{\mathrm{QCD}}$ as in the dipole cascade.

The whole procedure will now be as follows:

1. First the number of partons, $n \leq N$, to be generated is chosen according to the integrated jet rates $R_{n}$ from the tree-level MEs. Note that $\sum R_{n}$ is larger than 1 since we do not include virtual corrections. 
2. Then the momenta of the $n$ partons are generated according to the $\mathcal{O}\left(\alpha_{\mathrm{s}}^{n-2}\right)$ tree-level ME. Afterwards the invariant $p_{\perp}^{2}$ of the $n$ partons is checked, and if anyone is below $p_{\perp c}^{2}$, the state is rejected and the procedure is restarted at step 1 .

3. Now, all the intermediate states $S_{2}, \ldots, S_{n-1}$ and scales $p_{\perp 1}^{2}, \ldots, p_{\perp n-2}^{2}$ corresponding to a sequence of dipole emissions are reconstructed according to the algorithm in section 3 .

4. The generated event is rejected and we restart at step 1 with a probability given by equation (10).

5. We now make a trial emission with the dipole cascade from the state $S_{2}$ starting from the maximum scale limited by the center of mass energy. If this emission is at a scale above $p_{\perp 1}^{2}$, the event is rejected and we restart from step 1. If not, a trial emission is performed from the state $S_{3}$ with a maximum scale of $p_{\perp 1}^{2}$. If this emission is at a scale above $p_{\perp 2}^{2}$ the event is rejected and we restart from step 1. This procedure is repeated for all states down to $S_{n-1}$. If no rejection has been made, a trial emission is made from the ME-generated $n$-parton state starting from the scale $p_{\perp n-2}^{2}$. There are now two cases

- If $n=N$ the trial emission is always kept and the dipole cascade is allowed to continue down to the cutoff $p_{\perp c}^{2}$ and the event is accepted.

- If $n<N$, and all parton pairs pass the ME cut, $Q_{0}$, the event is rejected and we restart from step 1. If any of the partons fail the cut, the trial emission is accepted and the dipole cascade is allowed to continue down to the cutoff $p_{\perp c}^{2}$ and the event is accepted.

For a pictorial description of the procedure, figure 1 1 shows the regions in a symbolic two-dimensional phase space, in which the Sudakov veto algorithm is used in the case of $N=4$ and $n=2,3,4$. It is clear that if there is only one emission inside the $\mathrm{ME}$ cut, it is handled by the ME generator and this region is never populated by the dipole cascade. If there are two emissions inside the ME cut, the two hardest ones are always handled by the ME generator while additional emissions are given by the dipole cascade. In this way there is no double counting between the ME generator and the dipole cascade. Note that if the trial emission in e.g the $n=2$ case is outside the ME cut and therefore is accepted as shown in figure 2, further emissions inside the ME cut are allowed without any risk of double counting.

Although the procedure is to add a dipole cascade to an $N$-parton matrix element generator, the result is that all final multi-parton states are distrubuted as if generated by the dipole cascade, except that if the $n-2$ (with $n \leq N$ ) hardest emissions are inside the matrix element cut, their distribution is described by the exact tree-level matrix element.

More precisely, we have the following possible situations: 

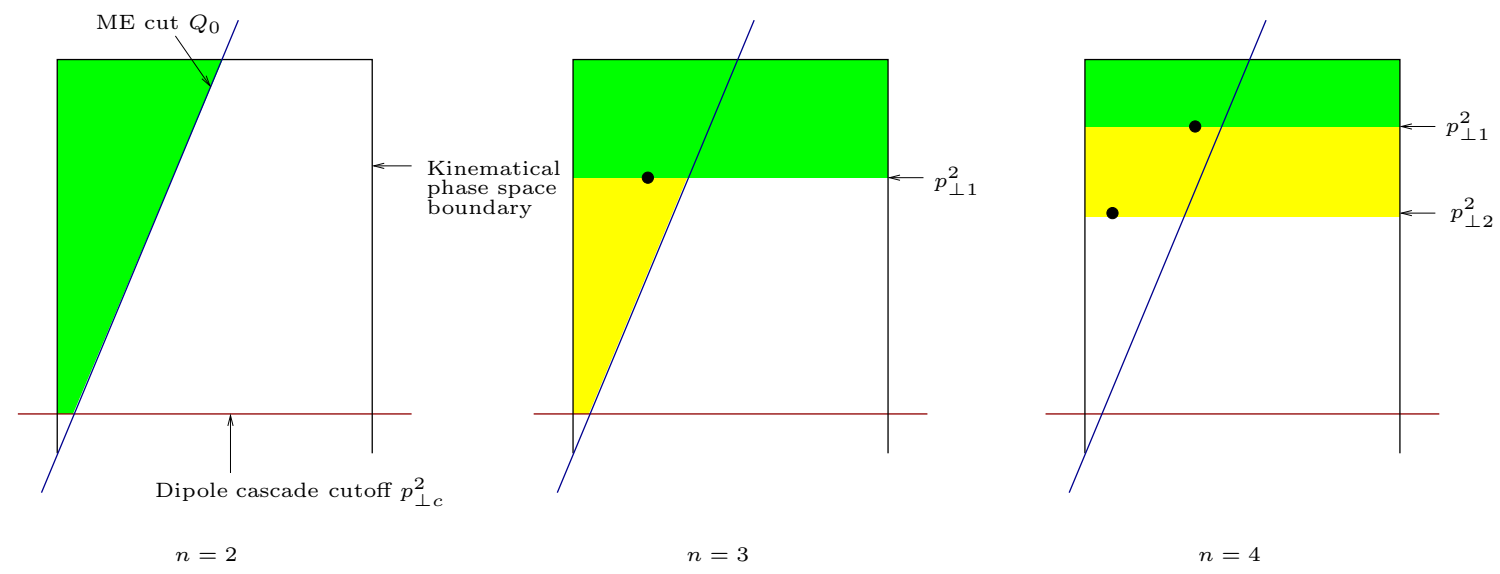

Figure 1: The integration regions (shaded) for the Sudakov form factors in a symbolic two-dimensional phase space, giving the probability to accept a ME-generated 2, 3 and 4 parton states in the case $N=4$. The evolution variable in the cascade is assumed to be along the vertical axis, while the horizontal axis is some rapidity or energy splitting variable. The cutoff in the ME is along the diagonal line.

- If the hardest emission is outside the ME cutoff, all emissions are given by the dipole splitting functions, even if softer emissions are inside.

- If the hardest emission is inside the ME cutoff, but the second hardest is outside, the hardest emission will be given by the ME, while all subsequent ones are given by the dipole splitting functions.

- If the two hardest emissions are inside the ME cutoff they are given by the $\mathrm{ME}$, while all subsequent emissions are given by the dipole splitting functions (irrespective of whether they are inside the cutoff or not).

In all cases the Sudakov form factors are the same irrespective of whether the emissions are given by the ME or the dipole splitting functions.

It is clear that there should only be a small dependence on the $Q_{0}$ since the only change when going outside the cut is that the emissions are governed by the leading logarithmic expressions rather than the exact ME and these should be very similar for a small enough cut. It is important that the extra $p_{\perp}$ cut used in step 2 is the same for the ME-generated state as for the subsequent dipole cascade, otherwise the ME may populate phase space outside of the reach of the cascade. This is, in fact, not quite trivially achieved in the cascade itself. The reason is that the recoils from an emission in one dipole may push the invariant $p_{\perp}$ of a parton in a neighboring dipole below the cutoff $p_{\perp c}$. To be completely consistent, the dipole cascade is therefore used with an extra cut vetoing emissions which push other partons below the cut. 

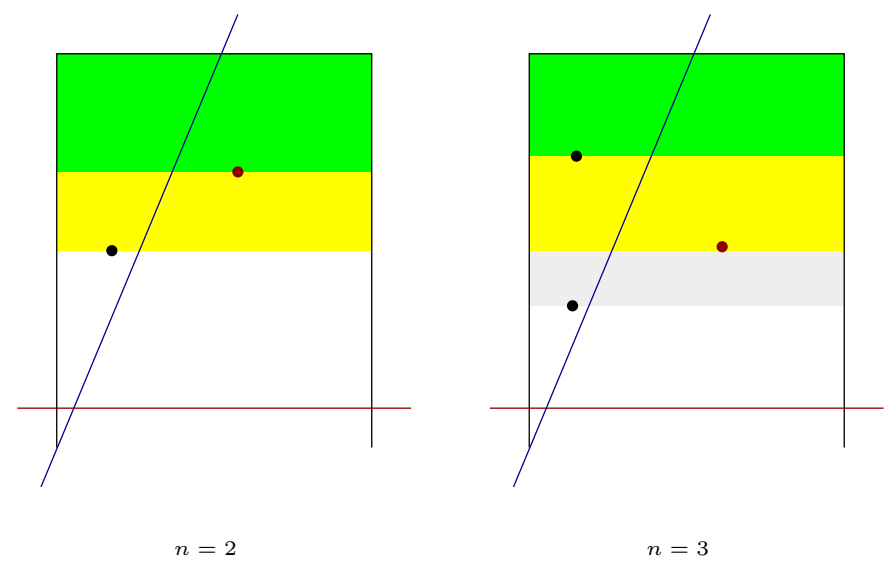

Figure 2: Possible sequences of dipole cascade emissions from $n=2$ and $n=3$ states generated with MEs.

\section{Results}

To check that the procedure works, the simplest thing is to test it for $N=3$ since the ARIADNE dipole cascade is already corrected to match the $\mathcal{O}\left(\alpha_{\mathrm{s}}\right)$ ME. And, indeed the new procedure completely agrees with standard ARIADNE in this case. A less trivial test is to look at the $N=4$ case. For this a modification of the $\mathcal{O}\left(\alpha_{\mathrm{s}}^{2}\right)$ ME generator implemented in PYTHIA is used, stripped down so that it only uses the tree-level MEs with a simple invariant mass cutoff. This is then used together with the new dipole cascade interface algorithm implemented in ARIADNE.

First we look at some standard event shapes. These are known to be very well described by the ARIADNE program (see eg. [32]). We will not directly compare to data since this would not be meaningful without a retuning of the hadronization parameters. Instead we will compare the new procedure with the standard ARIADNE program on parton level. Figure 3 shows the dependence on the ME cut $Q_{0}$ of some standard event shapes which should be sensitive to $\mathcal{O}\left(\alpha_{\mathrm{s}}^{2}\right)$ effects. The differences w.r.t. ARIADNE are very small and rather than showing the event shapes them selves, figure 3 shows the ratio to the ARIADNE results. The fact that the differences are so small is not a surprise. Already in [33] it was shown that the dipole cascade agrees very well with $\mathcal{O}\left(\alpha_{\mathrm{s}}^{2}\right)$ MEs in most regions of phase space. For large $Q_{0}$ the new procedure reduces to the standard ARIADNE program as expected. The dependence on the $Q_{0}$ is small but not zero. The largest difference is found i the D4 distribution which measures the smallest distance, according to the Durham jet-algorithm [24,25], between two jets when an event has been clustered to four jets. It is a bit worrying that the dependence does not seem to disappear even for very small $y_{0}=Q_{0}^{2} / E_{\mathrm{cm}}^{2}$. This may be expected for the D4 distribution when the distance between jets are smaller than $y_{0}$, but it is also present in the 
(a) Objateness normalized to Ariadne

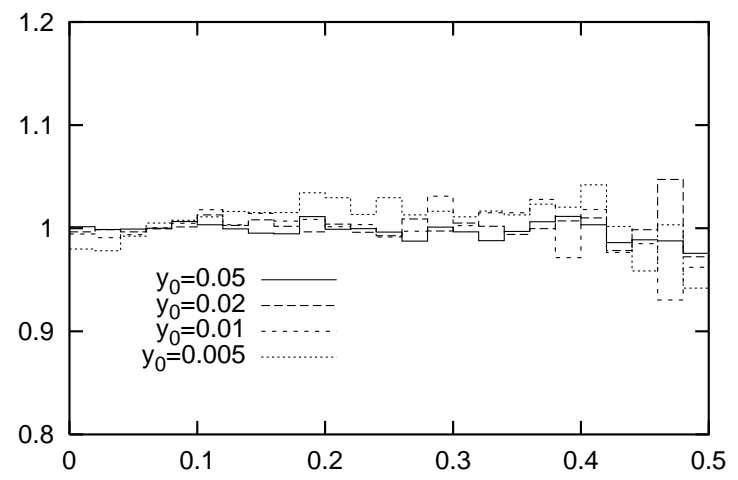

(c) $p_{\text {t out }}$ normalized to Ariadne

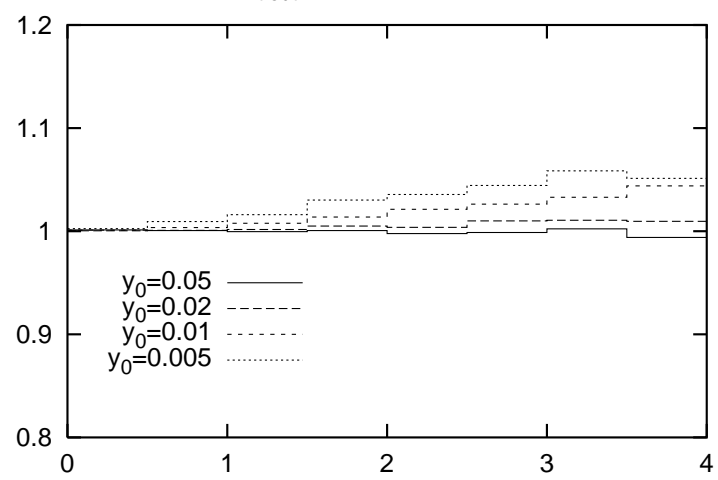

(b) D4 normalized to Ariadne

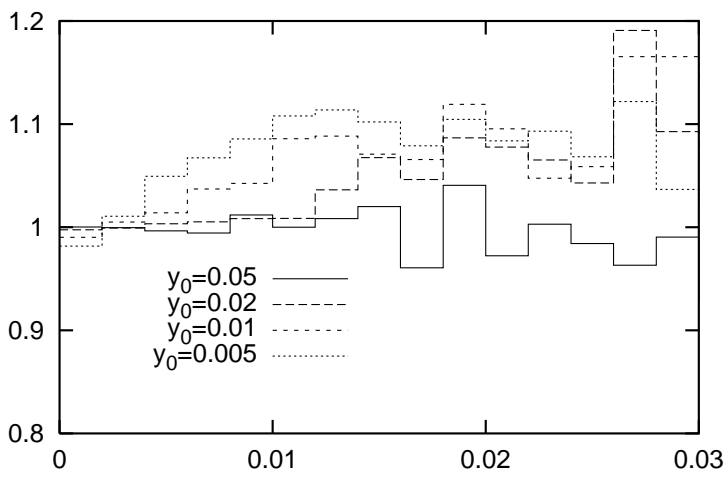

(d) $p_{\text {t out }}$ normalized to Ariadne (massless)

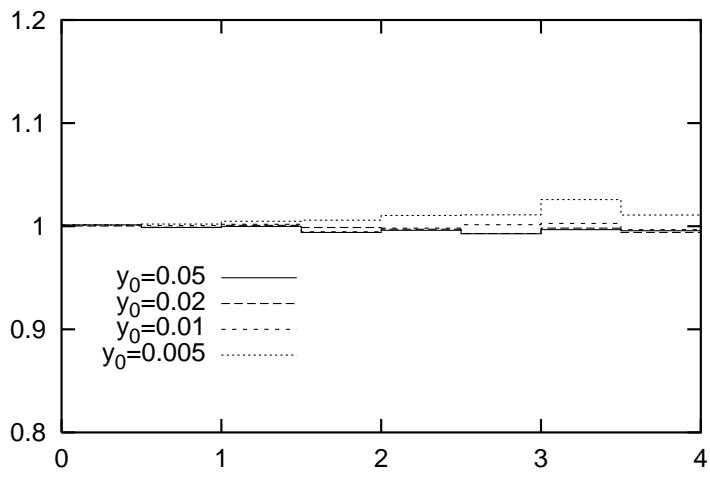

Figure 3: Ratios of parton level event shapes at $E_{\mathrm{CM}}=91$ GeV for the new $M E$ matching algorithm using different values of $Q_{0}$ w.r.t. to the standard ARIADNE program. The distributions are (a) oblateness, (b) 4-jet resolution value for the Durham jet algorithm and (c) $p_{\perp \text { out. }}$ (d) is the same as (c) but with massless quarks and no secondary quarks both for the new ME matching and for standard ARIADNE. In all cases, the full line is with $y_{0}=Q_{0}^{2} / Q^{2}=0.05$, long-dashed: $y_{0}=0.02$, dashed: $y_{0}=0.01$ and dotted: $y_{0}=0.005$.

$p_{\perp \text { out }}$ distribution even for fairly large values. The reason can be traced to the treatment of quark masses which is not exact in ARIADNE (nor is it exact in the tree-level matrix elements in PYTHIA). In addition there are some uncertainties in the treatment of secondary quarks in ARIADNE[. As seen in in figure $3 \mathrm{~d}$, if all quark masses are put to zero and secondary quarks are taken away, the dependency on $y_{0}$ becomes much smaller, but it still does not go away for really small values. A possible explanation may be that the dipole clustering routine has some problems for very small resolution scales as described in [28], but the dependence is in any case much smaller than the uncertainties due to hadronization parameters and the

\footnotetext{
${ }^{5}$ An investigation of the effects of quark masses and secondary quarks will be presented in a future publication 34
} 
(a) $\cos \theta_{\mathrm{BZ}}$

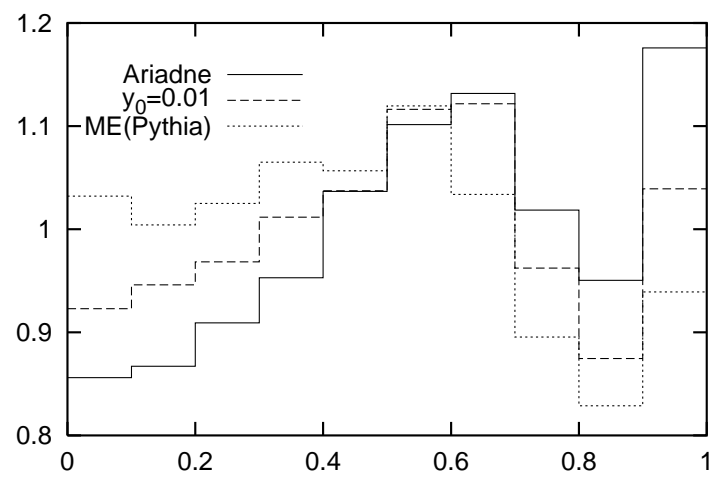

(b) $\cos \theta_{\mathrm{BZ}}$

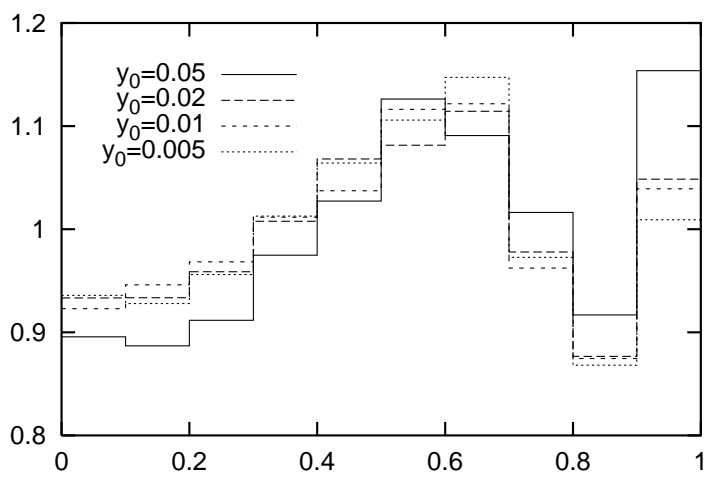

Figure 4: The distribution in the Bengtsson-Zerwas angle on parton level using the $J A D E$ jet algorithm 35] with $y_{\text {cut }}=0.03$. In (a) the full line is standard ARIADNE, the dashed line is the new ME matching algorithm with $y_{0}=0.01$ and the dotted line is the tree-level $\mathcal{O}\left(\alpha_{\mathrm{s}}^{2}\right)$ ME-only generator in PYTHIA with $y_{0}=0.01$. In (b) the full, long dashed, dashed and dotted lines are all the new ME matching algorithm with $y_{0}=0.05,0.02,0.01$ and 0.005 respectively.

basic parameters in the dipole cascade, $p_{\perp c}$ and $\Lambda_{\mathrm{QCD}}$.

To really see the influence of the ME matching one must look at details in the correlations between jets. Here we will look at Bengtsson-Zerwas angle [36], i.e. the angle between the plane spanned by the two largest jets and the plane spanned by the two smallest jets in a four-jet event, which is not at all described by the standard ARIADNE program. In figure 4 we see that the new matching procedure is closer than standard ARIADNE to the result from the pure $\mathcal{O}\left(\alpha_{\mathrm{s}}^{2}\right)$ ME generator in PYTHiA. It does not, and should not, exactly reproduce the pure ME approach since the correlation is smeared by the subsequent soft radiation.

\section{Conclusions and outlook}

The procedure presented here to correct the dipole cascade in ARIADNE to match the exact $\mathcal{O}\left(\alpha_{\mathrm{s}}^{N}\right)$ matrix elements works. The only additional parameter needed is the cutoff $Q_{0}$ in the ME generation, but the results have been shown to be fairly insensitive to the value chosen as long as it is reasonably small. Although the results presented here has only been for $N=4$, the procedure is completely general and can be applied to basically any $N$-parton $\mathrm{ME}$ generator. One advantage is that the procedure is practically non-intrusive with respect to the ME generator used. On the other hand the vetoing technique makes the generation very inefficient for very small $Q_{0}$. 
There are a few uncertainties in this algorithm, mainly to do with the reconstructions of the emissions. It is not quite clear what to do with reconstructed emissions which are not ordered. The procedure presented in section 3 is not unique and, in fact, several other options have been tested. The results are, however, rather stable w.r.t. such variations.

In any case the procedure will give multi parton final states where the first $N-2$ emissions are correct to exact $\mathcal{O}\left(\alpha_{\mathrm{s}}^{N-2}\right)$ accuracy and all others are correct to MLLA accuracy.

It could be possible to also include the exact virtual corrections to $\mathcal{O}\left(\alpha_{\mathrm{s}}^{N-2}\right)$. This would involve correcting the tree-level matrix elements with the difference between the exact and the leading logarithmic parts of the virtual plus unresolved terms, for the $N=4$ case effectively turning $R_{2}$ and $R_{3}$ in equation (8) into

$$
\begin{aligned}
R_{2}\left(\rho_{m}, \rho_{0}\right)= & \Delta_{S_{2}}\left(\rho_{m}, \rho_{0}\right)+\alpha_{\mathrm{s}} \delta C_{0,1}^{\mathrm{MEPS}}\left(\rho_{m}, \rho_{0}\right)+\alpha_{\mathrm{s}}^{2} \delta C_{0,2}^{\mathrm{MEPS}}\left(\rho_{m}, \rho_{0}\right), \\
R_{3}\left(\rho_{m}, \rho_{0}\right)= & \alpha_{\mathrm{s}} \int_{\rho_{0}}^{\rho_{m}} d \rho c_{1,1}^{\mathrm{ME}}\left(\rho_{m}, \rho\right) \times \\
& {\left[\Delta_{S_{2}}\left(\rho_{m}, \rho\right) \Delta_{S_{3}}\left(\rho, \rho_{0}\right)+\alpha_{\mathrm{s}} \delta C_{1,2}^{\mathrm{MEPS}}\left(\rho_{m}, \rho, \rho_{0}\right)\right] }
\end{aligned}
$$

where the difference between the full coefficients from the summed virtual and unresolved diagrams in the matrix element and the corresponding parton shower ones, from the expansion of the Sudakov form factors and the running of $\alpha_{\mathrm{s}}$ with transverse momenta in the parton cascade, are symbolically written $\delta c_{n, m}^{\mathrm{MEPS}}=$ $c_{n, m}^{\mathrm{ME}}-c_{n, m}^{\mathrm{PS}}$. These coefficients are free from singularities, but the cutoff in the matrix element generation must be chosen so that the differential $n$-jet rates will come out positive in the whole phase space. Further investigations is needed to see if this at all can be accomplished.

It should be noted that a procedure for combining next-to-leading order matrix element with parton showers has recently been proposed by Frixione and Webber [37]. However, such a generator will produce negatively weighted events and is therefore difficult to use as a general purpose event generator.

Finally it should be noted that it is possible to use the procedure presented in this paper also for collisions with incoming hadrons. The additional complication is that the reconstruction of the intermediate states and scales must take into account that there may both be initial- and final-state emissions, where the former depends on the parton density distributions of the incoming hadrons. But otherwise the procedure would be the same: Use a tree-level matrix element generator to generate lowest order and subsequent higher order partonic states, reconstruct the possible emission histories according to a parton cascade scheme together with the corresponding emission scales, calculate the running of $\alpha_{\mathrm{s}}$, calculate the Sudakov 
form factors with the veto algorithm using trial emissions from the reconstructed states, and continue the cascade.

\section{References}

[1] G. Gustafson and U. Pettersson Nucl. Phys. B306 (1988) 746.

[2] L. Lönnblad Comput. Phys. Commun. 71 (1992) 15-31.

[3] M. Bengtsson and T. Sjostrand Phys. Lett. B185 (1987) 435.

[4] M. Bengtsson and T. Sjöstrand Nucl. Phys. B289 (1987) 810.

[5] M. H. Seymour Nucl. Phys. B436 (1995) 443-460, hep-ph/9410244.

[6] M. H. Seymour Comp. Phys. Commun. 90 (1995) 95-101, hep-ph/9410414.

[7] M. H. Seymour, "Matrix element corrections to parton shower simulation of deep inelastic scattering." Contributed to 27th International Conference on High Energy Physics (ICHEP), Glasgow, Scotland, 20-27 Jul 1994.

[8] L. Lönnblad Z. Phys. C65 (1995) 285-292.

[9] L. Lönnblad Nucl. Phys. B458 (1996) 215-230, hep-ph/9508261.

[10] G. Miu and T. Sjöstrand Phys. Lett. B449 (1999) 313-320, hep-ph/9812455.

[11] S. Mrenna, "Higher order corrections to parton showering from resummation calculations," hep-ph/9902471.

[12] G. Corcella and M. H. Seymour Nucl. Phys. B565 (2000) 227-244, hep-ph/9908388.

[13] J. Andre and T. Sjöstrand Phys. Rev. D57 (1998) 5767-5772, hep-ph/9708390.

[14] E. Norrbin and T. Sjöstrand Nucl. Phys. B603 (2001) 297-342, hep-ph/0010012.

[15] B. Pötter Phys. Rev. D63 (2001) 114017, hep-ph/0007172.

[16] S. Catani, F. Krauss, R. Kuhn, and B. R. Webber, "QCD matrix elements + parton showers," hep-ph/0109231.

[17] J. C. Collins JHEP 05 (2000) 004, hep-ph/0001040.

[18] Y. Chen, J. C. Collins, and N. Tkachuk JHEP 06 (2001) 015, hep-ph/0105291.

[19] G. Gustafson Phys. Lett. B175 (1986) 453.

[20] T. Sjöstrand, et al. Comput. Phys. Commun. 135 (2001) 238-259, arXiv:hep-ph/0010017. 
[21] A. H. Mueller Nucl. Phys. B213 (1983) 85.

[22] A. H. Mueller Nucl. Phys. B241 (1984) 141.

[23] Y. L. Dokshitzer and S. I. Troian. LENINGRAD-84-922.

[24] Y. L. Dokshitzer. in Workshop on Jet studies at LEP and HERA, Durham 1990, see J. Phys. G17 (1991) 1572ff.

[25] S. Catani, Y. L. Dokshitzer, M. Olsson, G. Turnock, and B. R. Webber Phys. Lett. B269 (1991) 432-438.

[26] G. Corcella et al. JHEP 01 (2001) 010, hep-ph/0011363.

[27] L. Lönnblad Z. Phys. C58 (1993) 471-478.

[28] S. Moretti, L. Lönnblad, and T. Sjöstrand JHEP 08 (1998) 001, hep-ph/9804296.

[29] B Andersson, G. Gustafson, L. Lönnblad Nucl. Phys. B 339 (1990) 393.

[30] E. Boos et al. hep-ph/0109068.

[31] R. Kleiss Phys. Lett. B180 (1986) 400.

[32] K. Hamacher and M. Weierstall, "The Next Round of Hadronic Generator Tuning Heavily Based on Identified Particle Data," hep-ex/9511011.

[33] B. Andersson, G. Gustafson, and C. Sjögren Nucl. Phys. B380 (1992) 391-407.

[34] L. Lönnblad and C. Ortiz. Preprint in preparation.

[35] JADE Collaboration, W. Bartel et al. Z. Phys. C33 (1987) 339.

[36] M. Bengtsson and P. M. Zerwas Phys. Lett. B208 (1988) 306.

[37] S. Frixione and B. R. Webber hep-ph/0204244. 